\title{
OSTEOGENESIS IN THE OSTEOARTHRITIC FEMORAL HEAD*
}

\author{
A Study Using Radioactive ${ }^{32} \mathbf{P}$ and Tetracycline Bone Markers
}

\section{A. K. Jeffery, Dunedin, New Zealand}

From the Department of Orthopaedic Surgery, University of Otago Medical School, Dumedin

Remodelling of bone structure is a distinctive feature of osteoarthritis, particularly in its more advanced stages. Continuing processes of bone resorption and bone regeneration may gradually alter the shape of adjacent articular surfaces sufficiently to impair joint function.

Although the pathogenesis of these bone changes is poorly understood, they appear to be influenced by an alteration in mechanical forces within the diseased joint (Collins 1949). In the osteoarthritic femoral head, degeneration and loss of cartilage over the major weightbearing segment is followed by reactive sclerosis and cyst formation in the underlying bone. Peripherally, and in areas subjected to less compression force, osteophytic outgrowths are commonly seen (Fig. 1).

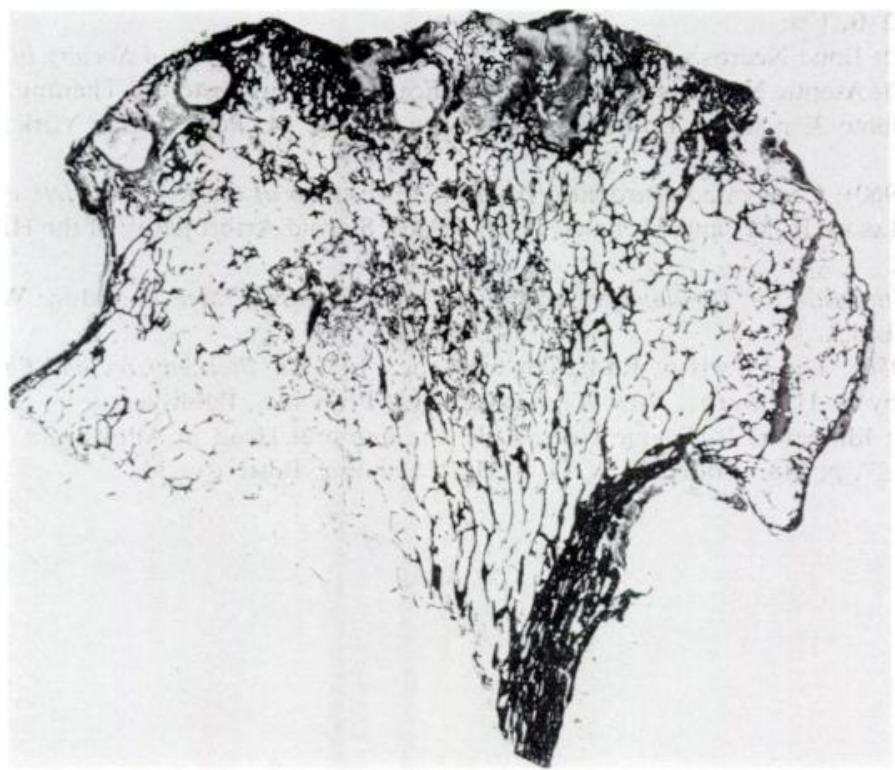

FIG. 1

Section through a femoral head showing the changes in advanced osteoarthritis. Bone sclerosis and cysts are seen in the superior weightbearing segment and a large medial osteophyte is present.

In the present study, remodelling and regeneration of bone in the osteoarthritic femoral head have been examined using radioactive ${ }^{32} \mathrm{P}$ and tetracycline as in vivo bone markers. Radioactive ${ }^{32} \mathrm{P}$ is incorporated in areas of new-formed bone, where it is fixed in a stable form and can be identified by autoradiographic means (Leblond, Wilkinson, Bélanger and Robichon 1950). Using tracer doses, the macroscopic uptake of ${ }^{32} \mathrm{P}$ within the femoral head

* Based on a paper presented at the Annual Meeting of the New Zealand Orthopaedic Association, Rotorua, September 28, 1971. 
could be examined. Tetracycline was used as it is also incorporated in new bone, and its distribution in the skeleton is similar to that of the bone-seeking radioisotopes (Ibsen and Urist 1964). Using techniques of ultraviolet photography and ultraviolet microscopy, tetracycline uptake could be studied and examined both macroscopically and microscopically.

\section{MATERIAL}

Fifty femoral heads were studied. Forty-six specimens were removed from patients undergoing total hip replacement for advanced osteoarthritis and four were obtained from two patients who died from severe intracranial haemorrhage. Before removal of the femoral head each patient was given tetracycline and/or ${ }^{32} \mathrm{P}$ (Table I). Radioactive ${ }^{32} \mathrm{P}(250 \mu \mathrm{Ci})$ was given intravenously two hours before operation, and tetracycline was given orally 1 gramme daily for five days and stopped twenty-four hours before operation. In ten patients two courses of tetracycline were given at varying intervals to provide double labelling of the bone.

\section{METHODS}

Each femoral head was cut in a coronal plane into serial slices approximately $0.4 \mathrm{~cm}$ thick. A representative slice, usually from the central segment of the femoral head. was decalcified and whole-head sections of $10-12 \mu$ were prepared using a standard histological

TABLE I

ANALysis of MATERIAL

\begin{tabular}{|c|c|}
\hline Bone label given pre-operatively & $\begin{array}{c}\text { Number of } \\
\text { femoral heads }\end{array}$ \\
\hline Radioactive ${ }^{32} \mathrm{P}$ & 14 \\
\hline Tetracycline (single course) & 15 \\
\hline Tetracycline (double course) & 10 \\
\hline Radioactive ${ }^{3 \cdot} \mathrm{P}$ and tetracycline & 11 \\
\hline Total & 50 \\
\hline
\end{tabular}

technique of double embedding with 2 per cent celloidin and wax. After staining with haematoxylin and eosin, each section provided a representative cross-section of the femoral head showing details of topography and changes in bone architecture (Fig. 1). Adjacent bone slices from the same femoral head were then examined for uptake of the bone markers. Contact autoradiography was used to localise areas of ${ }^{32} \mathrm{P}$ uptake macroscopically while ultraviolet fluorescent techniques were used to study tetracycline uptake both macroscopically, and microscopically.

Contact autoradiography - Using a technique described by Boyd and Calandruccio (1963) the bone slice to be examined was covered by a sheet of thin plastic and held in close contact with fine-grain industrial radiographic film (Kodak Microtex) in a screw clamp. The clamp was sealed in a lightproof box, and after storage in a deep freeze for four weeks, the film was removed and processed.

Tetracycline fluorescence-Areas of tetracycline uptake showed as a characteristic yellow fluorescence when the bone slice was examined under ultraviolet light. The fluorescence was recorded on colour film using multiple flashlight exposures with a Kodak Wratten 18A filter in front of the light source, and a Kodak Wratten $2 \mathrm{~A}$ filter over the camera lens. 
For microscopic study undecalcified sections of bone from varying sites in the femoral head were prepared using the method of Ball (1957). Each section (approximately $8 \mu$ ) was examined for tetracycline fluorescence using a Reichert "Binolux" microscope with a high pressure mercury-vapour light source, an E4 exciter filter and an Spl absorption filter. The section was then demounted, stained for bone mineral using the von Kossa technique and then remounted to allow further examination by ordinary light microscopy. In this way the same bone section was studied both for tetracycline uptake and for distribution of bone mineral.

\section{RESULTS}

Gross morphology-Typical changes of advanced osteoarthritis were seen in forty-six femoral heads (Fig. 1). Two specimens showed early osteoarthritic changes and two were normal. In forty-four of the specimens, articular cartilage was absent over the principal weight-bearing segment, exposing a shining surface of sclerotic bone pitted by small holes. Many of these

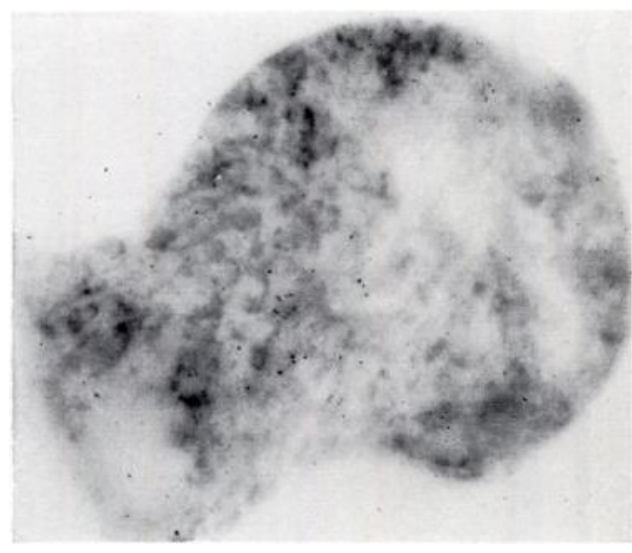

FIG. 2

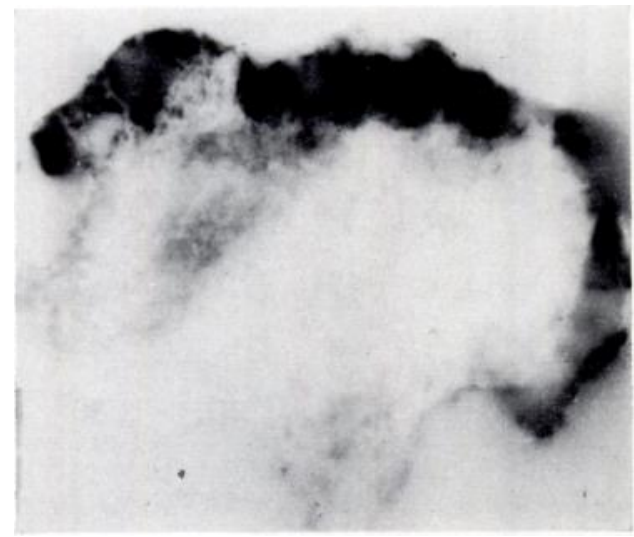

Fig. 4

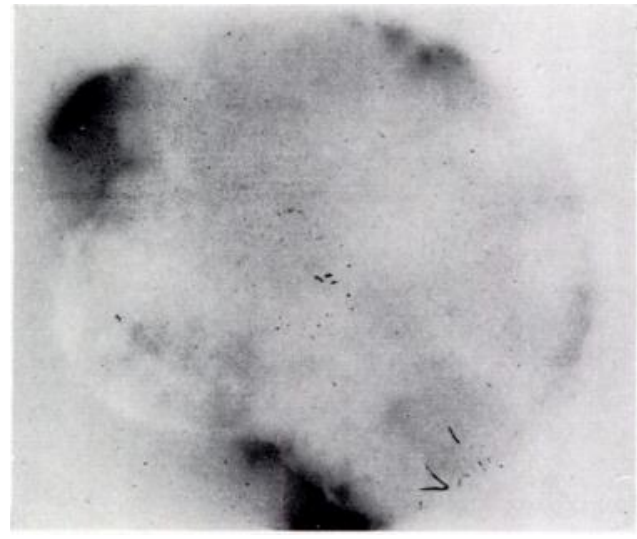

FIG. 3

Figs. 2 To 4

Autoradiographs showing radioactive ${ }^{3:} \mathrm{P}$ uptake in bone slices from normal and osteoarthritic femoral heads. Figure 2-A slice from the normal femoral head shows a relatively diffuse uptake of radioactive ${ }^{32} \mathbf{P}$. Figure 3 - In a slice from a case of early osteoarthritis, increased uptake of radioactive ${ }^{32} \mathrm{P}$ is seen in the peripheral osteophytes and in an area of bone adjacent to the degenerating cartilage. (Figures 6 and 7 show both tetracycline uptake and histological detail in the same bone slice.) Figure 4-In advanced osteoarthritis, intense uptake is seen in the superior segment of the femoral head as well as in the medial osteophyte. (Figure 1 shows histological detail in the same bone slice.)

holes communicated with subarticular cysts and they often contained fibrous tissue which projected beyond the bone surface. Histological preparation frequently caused shrinkage of this fibrous tissue. Bone cysts, when small, lay superficially and were localised to the pressure segment. Larger cysts extended more deeply in the femoral head and occasionally involved bone beneath articular cartilage. 
Osteophytes were seen in forty-eight specimens. These were invariably present peripherally at the junction of articular cartilage and synovium and less frequently at the attachment of the ligament of the femoral head. A constant finding in more advanced cases was a broad osteophyte applied to the medial aspect of the femoral head and lying adjacent to the fossa of the acetabulum. In addition to growing downwards, this broad osteophyte often extended upwards covering the original articular cartilage. The size of this osteophyte appeared to be related to the degree of flattening of the femoral head, supporting the observation of Harrison. Schajowicz and Trueta (1953) that displacement of the femoral head in the acetabulum acts as a stimulus to growth of osteophytes in the medial non-pressure area.

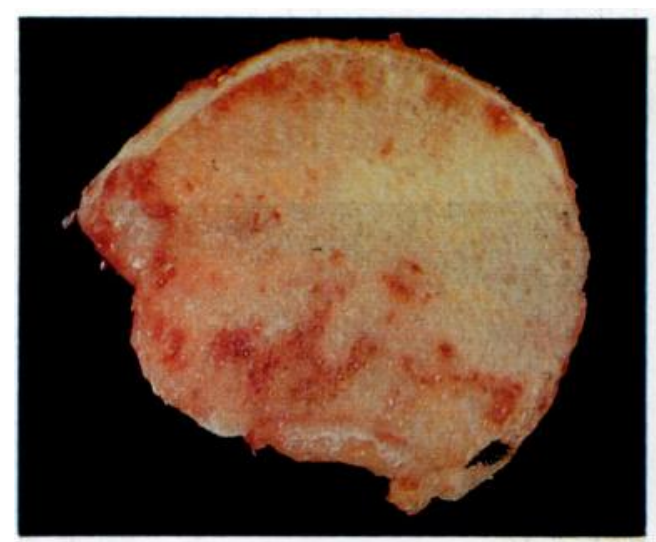

FIG. 5

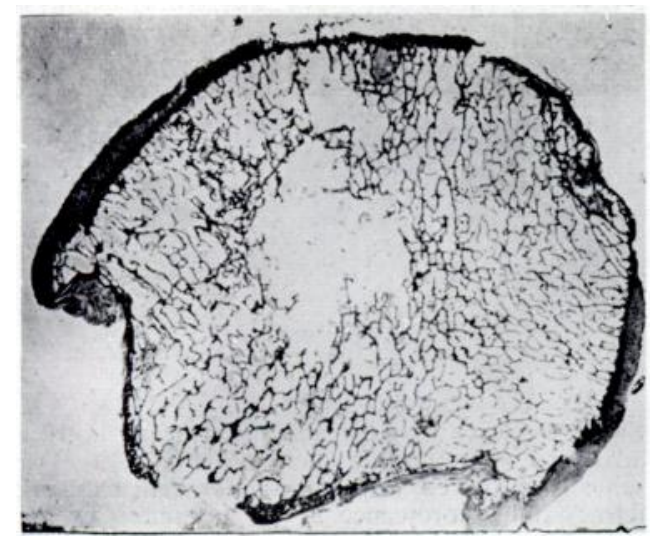

FIG. 7

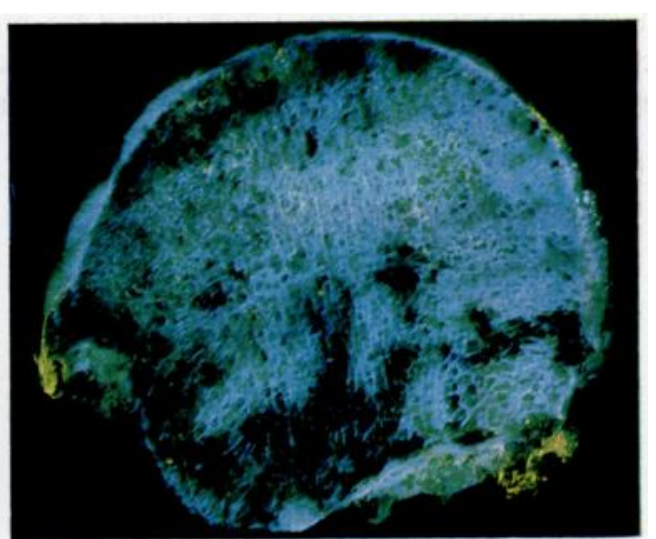

FiG. 6

Figs. 5 To 7

Figure 5-Slice from a femoral head showing early osteoarthritic changes. Figure 6-The same bone slice viewed with ultra-violet light, showing tetracycline fluorescence in the peripheral osteophytes. (Radioactive ${ }^{32} \mathrm{P}$ uptake in the same bone slice is shown in Figure 3.) Figure 7-Histological section from the same slice. (Haematoxylin and eosin.)

\section{AUTORADIOGRAPHY}

Bone slices from the two normal femoral heads showed a diffuse uptake of ${ }^{32} \mathrm{P}$ (Fig. 2). This diffuse reaction is produced by exchangeable or "labile" phosphate of the bone which is in continuous exchange with the phosphate ions of the blood. This differs from the "stable" phosphate which is fixed in areas of new bone formation and shows as a localised and persistent reaction on the autoradiograph (Leblond and colleagues 1950). Specimens with early changes of osteoarthritis showed an increased uptake of radioactive ${ }^{32} \mathrm{P}$ which appeared on the developed film as areas of intense reaction or "hot-spots". Greatest uptake was seen in osteophytes and in some areas of bone adjacent to degenerating articular cartilage (Fig. 3). In advanced osteoarthritis intense uptake of ${ }^{32} \mathrm{P}$ was noted in osteophytes and in the superior segment of the head where changes of cystic degeneration and bone sclerosis were greatest (Fig. 4).

VOL. 55 B, O. 2, MAY 1973 


\section{TETRACYCLINE FLUORESCENCE}

Macroscopic examination of bone slices under ultraviolet light showed the distribution of tetracycline was similar to that of the radioactive ${ }^{32} \mathrm{P}$, with uptake predominantly in osteophytes, around bone cysts and in some areas of bone sclerosis (Figs. 5 to 10). The areas of bone with greatest uptake of tetracycline were studied in greater detail by ultraviolet and standard microscopy of undecalcified sections.

Osteophytes-Osteophytes appear to arise and grow by a form of enchondral ossification (Collins 1949). Cells of cartilage or fibrous tissue differentiate and as the cells degenerate, their intercellular matrix is mineralised. At the growing surface of the osteophyte, the advancing zone of mineralisation showed intense tetracycline fluorescence (Figs. 11 to 14). More deeply in the osteophyte, bony trabeculae were remodelled by processes of bone resorption and regeneration. New bone in this situation was applied to existing trabeculae (appositional bone) and, in some areas, lay deep to a seam of osteoid tissue and osteoblasts.

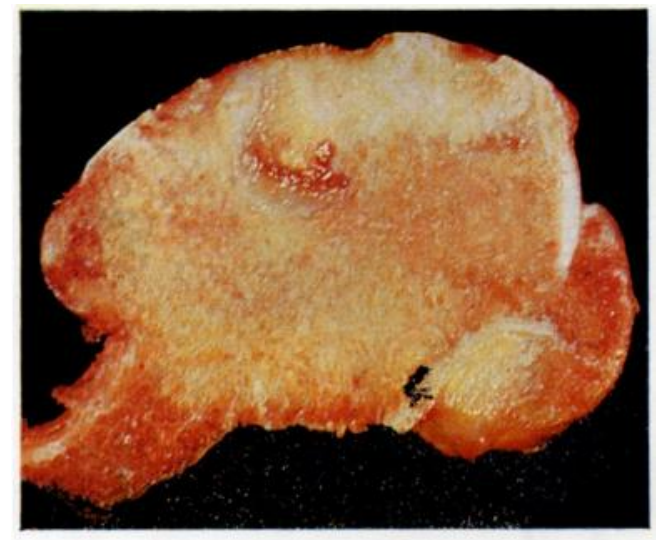

Fig. 8

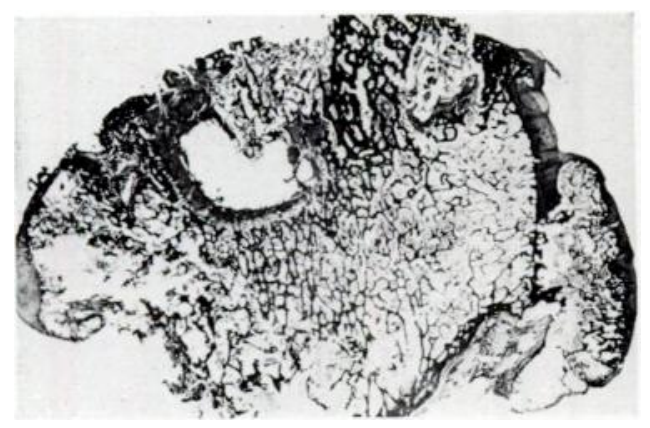

FIG. 10

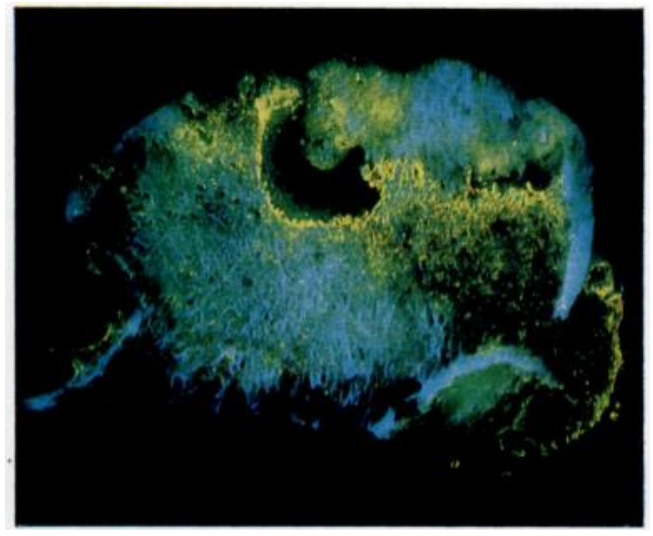

FIG. 9

Figs. 8 To 10

Figure 8-Slice through a femoral head showing advanced osteoarthritic changes. Figure 9-The same slice viewed with ultra-violet light showing tetracycline fluorescence at the periphery of the medial osteophyte and surrounding areas of bone degeneration and cyst formation. Figure 10Histological section from the same slice. (Haematoxylin and eosin.)

Bone sclerosis-Single and double labelling with tetracycline showed a continuous alteration in the trabecular system within the femoral head, particularly in the superior weight-bearing segment (Figs. 15 and 16). Subchondral bone lying deep to degenerating articular cartilage in this area was thickened by layers of appositional new bone. Tetracycline uptake was also seen in new bone advancing into the deeper layers of degenerating cartilage (Figs. 17 and 18). Appositional new bone showing intense tetracycline fluorescence was sometimes but not invariably seen lying deep to a prominent layer of osteoid tissue and osteoblasts (Figs. 19 and 20). 


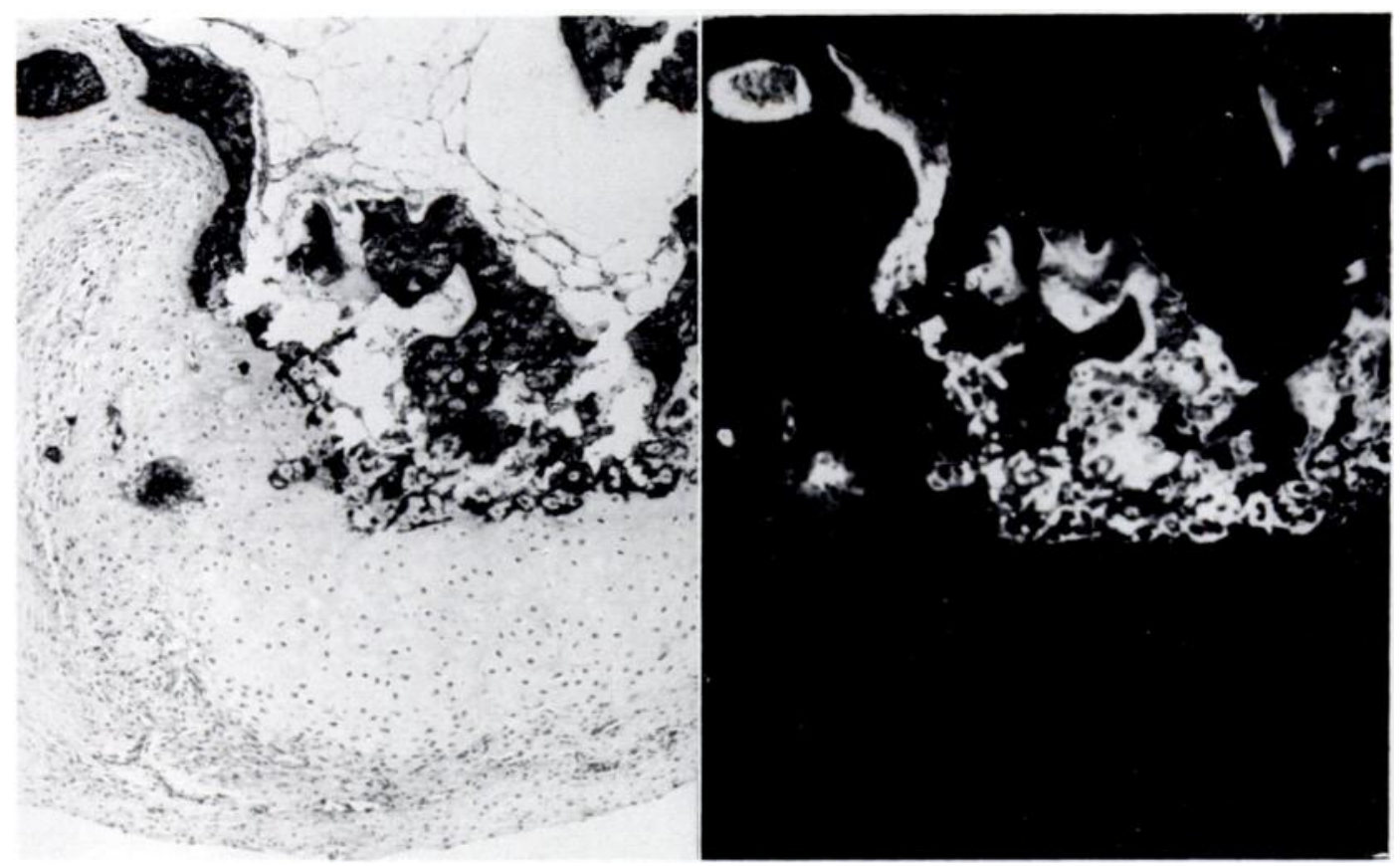

Fig. 11

FIG. 12

Figure 11-Undecalcified section from the tip of an osteophyte showing new bone advancing into an area of fibrous tissue that has undergone cartilaginous metaplasia. (Von Kossa stain. $\times 65$.) Figure $12-$ The same section viewed with ultra-violet light before staining, showing intense tetracycline fluorescence in the newly formed bone. $(\because 65$.)

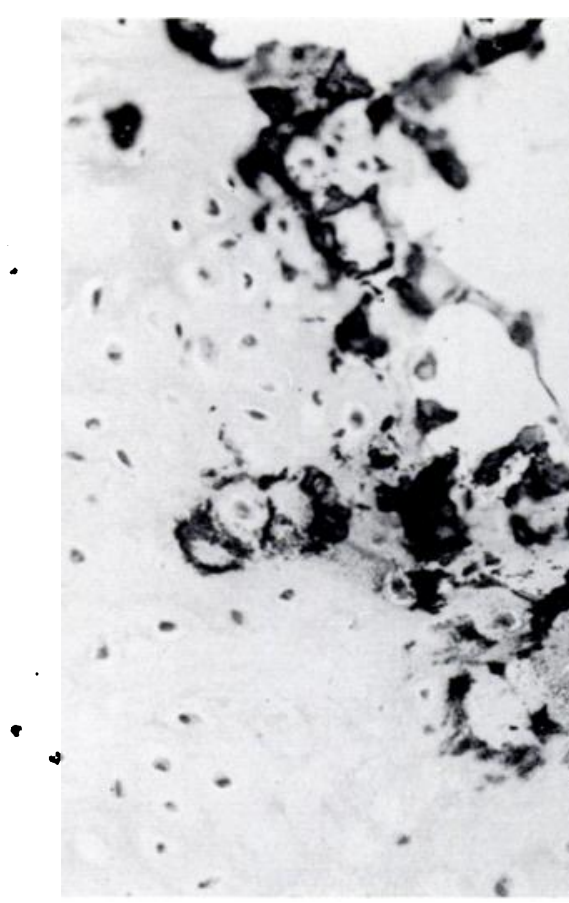

FIG. 13

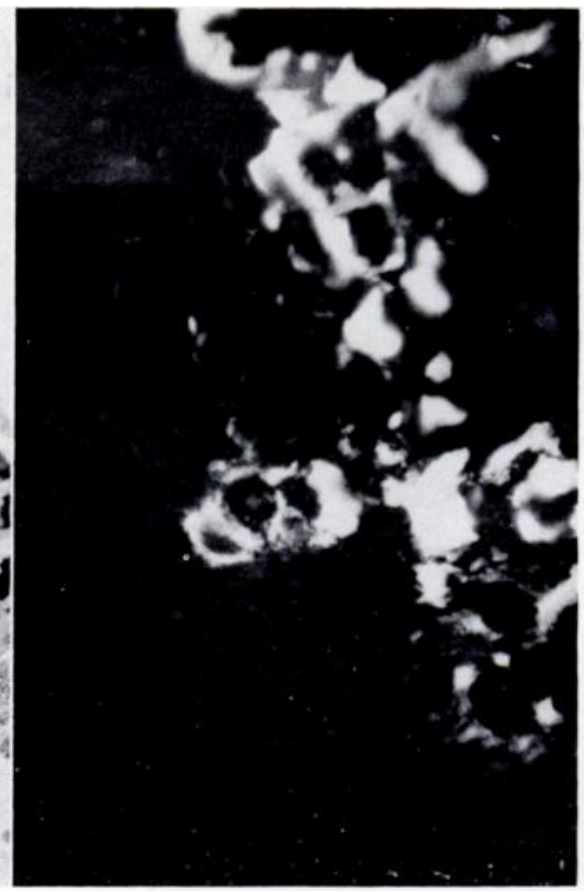

FIG. 14

Figure 13-High power view of the section in Figures 11 and 12, showing mineralisation of the intercellular matrix. (Von Kossa stain, $\times 220$.) Figure 14-The same section as in Figure 13 viewed with ultra-violet light before staining, showing tetracycline fluorescence in the newly formed bone mineral. $(\because 220$.) 
Bone cysts-Cyst walls were the site of considerable osteogenic activity. New bone formation was predominantly of the appositional type resulting in an increased thickness of existing trabeculae (Figs. 21 and 22).

In several sections, however, the fibrous tissue content of the cyst was undergoing cellular differentiation and a form of enchondral new bone formation was occurring. resembling that seen at the growing surface of the osteophyte (Fig. 23).

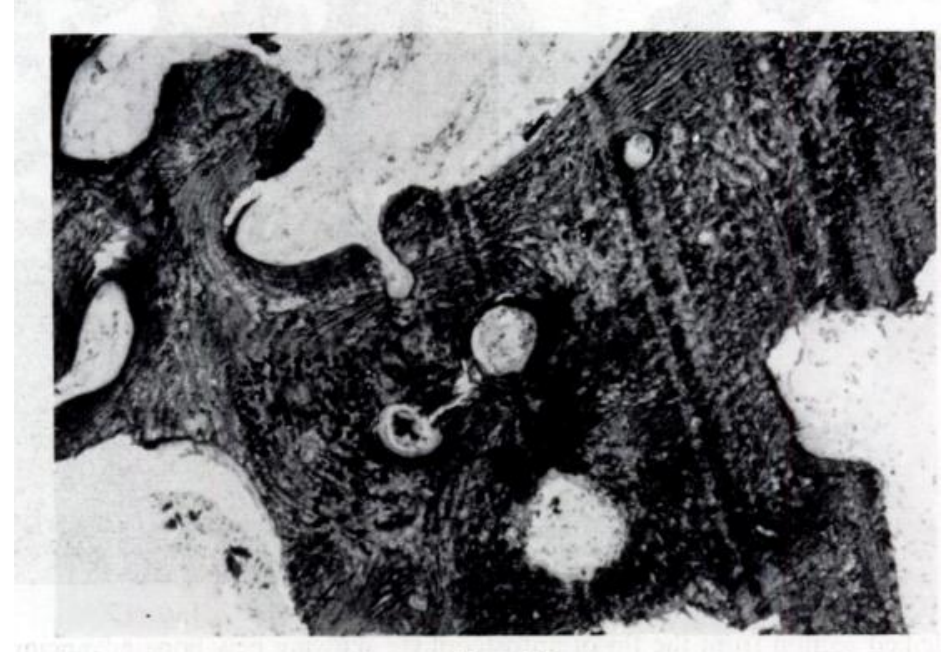

FiG. 15

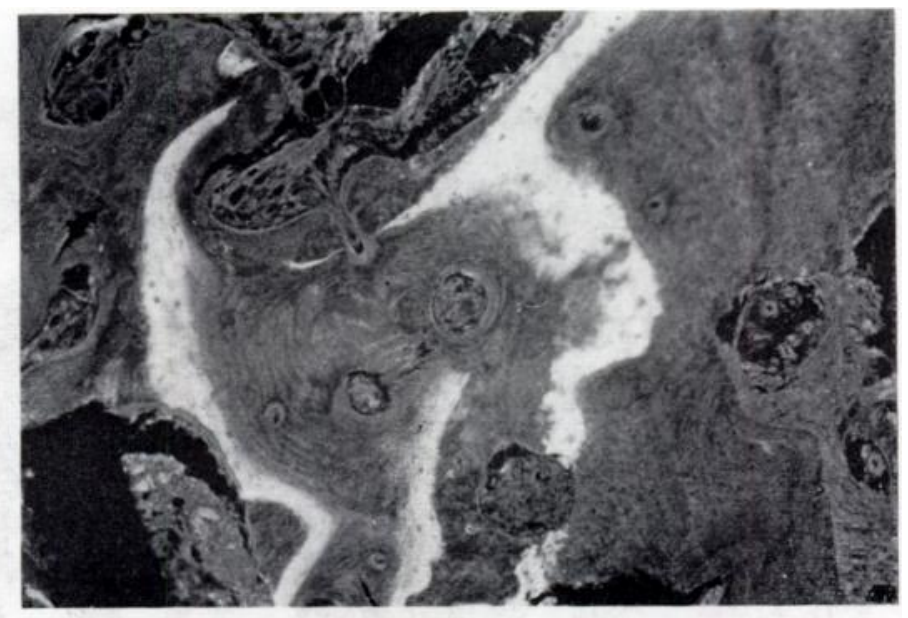

FiG. 16

Figure 15- Section of undecalcified bone from the superior weightbearing segment of the femoral head showing thickened bone trabeculae. (Von Kossa stain, $\times 60$.) Figure 16-The same section as in Figure 15 before staining and viewed with ultra-violet light. Tetracycline was given eight weeks before operation and tetracycline fluorescence situated deeply in the bone indicates the bone remodelling that has occurred since. $(\times 60$.

\section{DISCUSSION}

Previous studies have suggested that considerable bone activity is present in advanced osteoarthritis. External bone scanning techniques using the isotopes Ca47 and Sr85 show an increased rate of bone-tissue turnover in osteoarthritic joints (Bauer and Smith 1969). Batra and Charnley (1969) described the presence of osteoid tissue in the osteoarthritic femoral head and noted that it was localised mainly in the site of greatest degeneration in the pressure 
segment of the head. In the present study, fluorescence was prominent in bone deep to these osteoid seams (Figs. 19 and 20). The presence of osteoid in this situation appears to indicate an area of rapid appositional bone formation where mineralisation cannot keep pace with osteoid production.

Investigation of the vascular pattern in the osteoarthritic femoral head by Harrison and colleagues (1953), showed a state of hypervascularity as compared with the normal. This was greater in more advanced stages of the disease and was most marked in the pressure segment of the head, and also in relation to osteophytes and surrounding bone cysts. These are areas that show maximal osteogenic activity with increased uptake of radioactive ${ }^{32} \mathrm{P}$ and tetracycline.

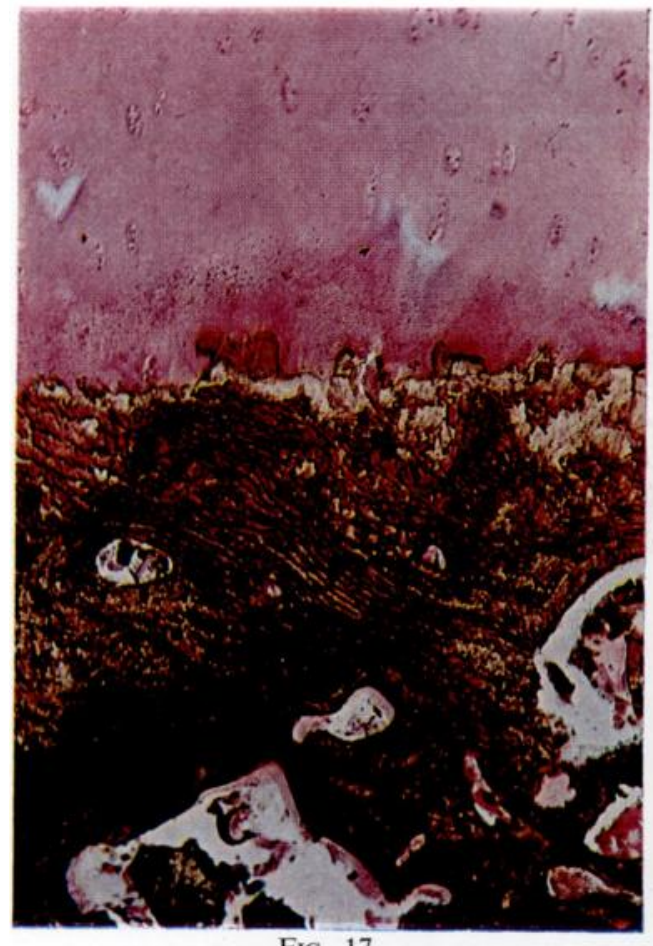

FIG. 17

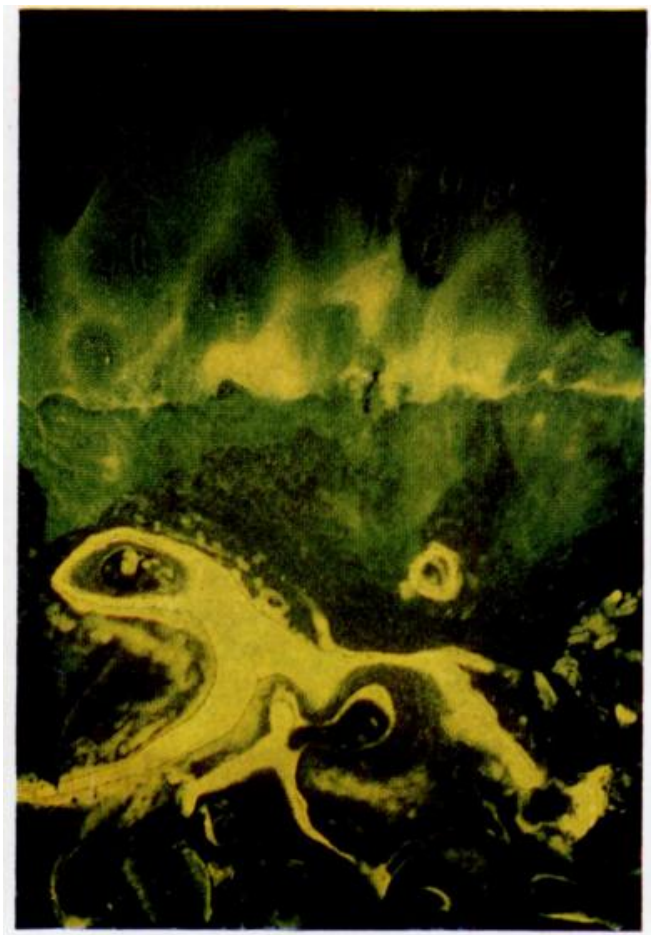

Fig. 18

Figure 17-Undecalcified section showing degenerating articular cartilage and thickened subchondral bone. Osteoid seams adjacent to bone are seen in the lower part of the section. (Von Kossa stain, $\times 75$.) Figure 18-The same section viewed with ultra-violet light before staining. Double labelling with tetracycline with eight-week interval shows the appositional bone formation and remodelling that has occurred. Tetracycline fluorescence is also prominent where new bone is penetrating the basal layer of articular cartilage. $(\times 75$.

Bone regeneration in the osteoarthritic head is of two types. There is a form of enchondral ossification in which the intercellular matrix of cartilage or differentiating fibrous tissue is mineralised and converted into bone. In the second type-appositional ossification-new bone is applied to an existing bone surface. Similar processes occur in the growing long bone, in which enchondral ossification is mainly concerned with growth in length and appositional ossification with increase of circumference.

In spite of the active osteogenesis apparent in advanced osteoarthritis, the repair of bone is usually inadequate and poorly coordinated. Osteophytes may alter joint congruity and impair movement, and in areas of bone sclerosis, trabeculae may be so thickened that osteocytes in the centre are deprived of nutrition and die. Bone regeneration is prominent in many areas around bone cysts but this is usually appositional bone causing sclerosis. In some areas, however, an attempt to obliterate the cyst by a form of enchondral ossification is seen.

VOL. 55 B, NO. 2, MAY 1973 


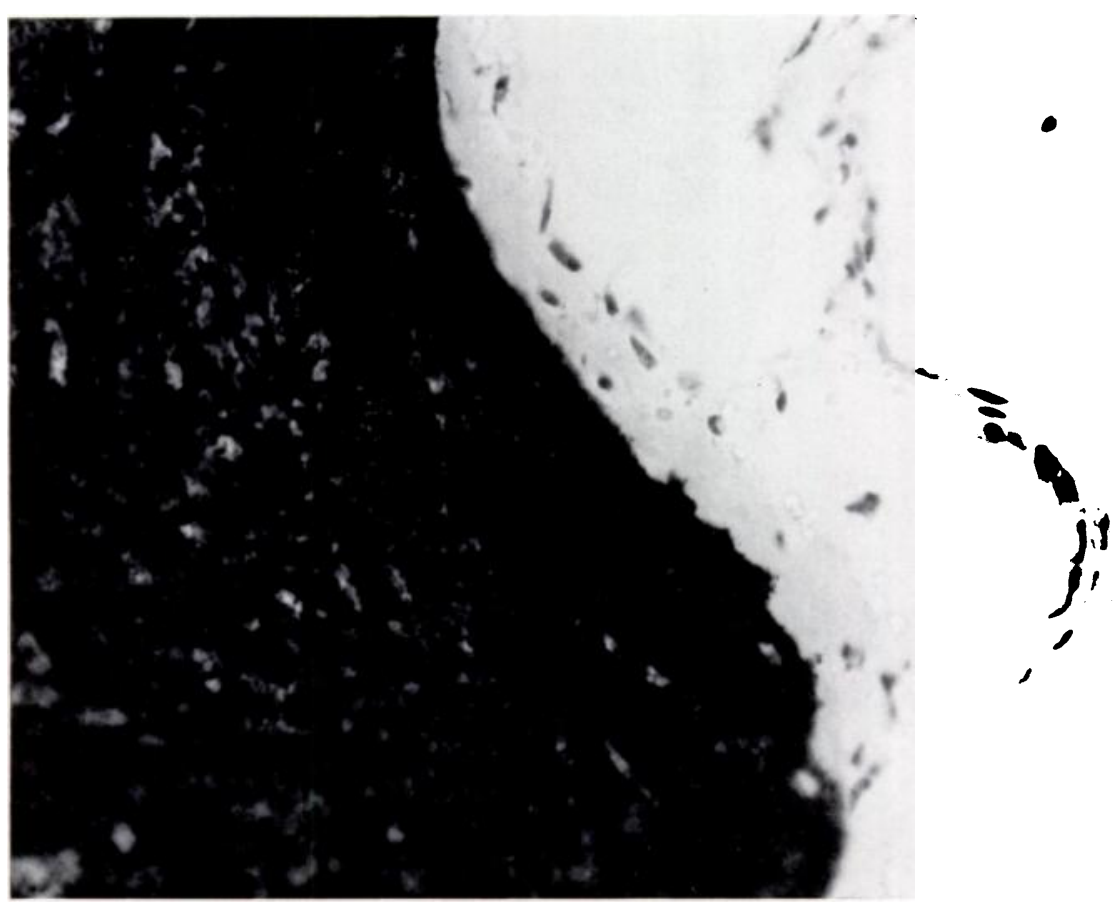

FIG. 19

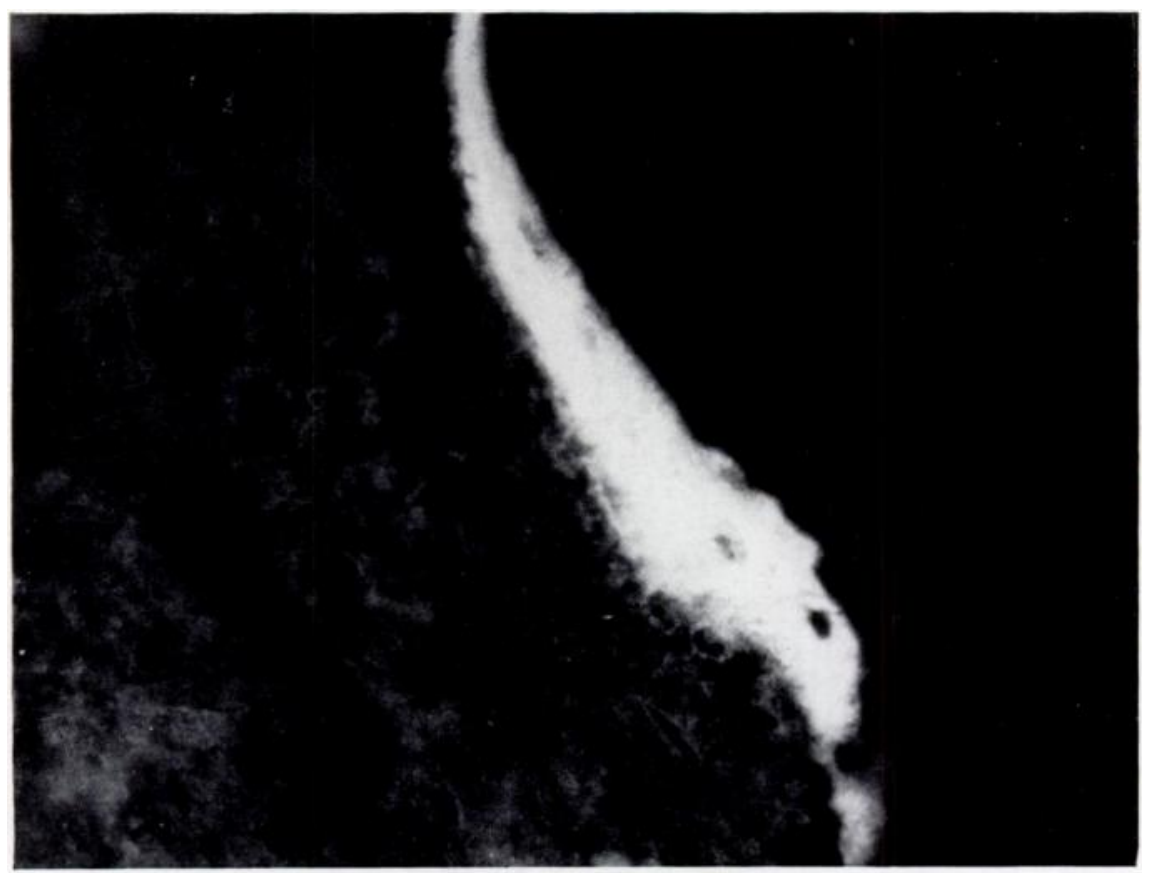

FIG. 20

Figure 19-Undecalcified section of bone showing a prominent osteoid seam with osteoblasts. (Von Kossa stain, $\times 360$.) Figure 20 -The same section as in Figure 19 viewed with ultra-violet light before staining. Intense tetracycline fluorescence is seen in bone lying immediately deep to the osteoid seam. $(\times 360$. 


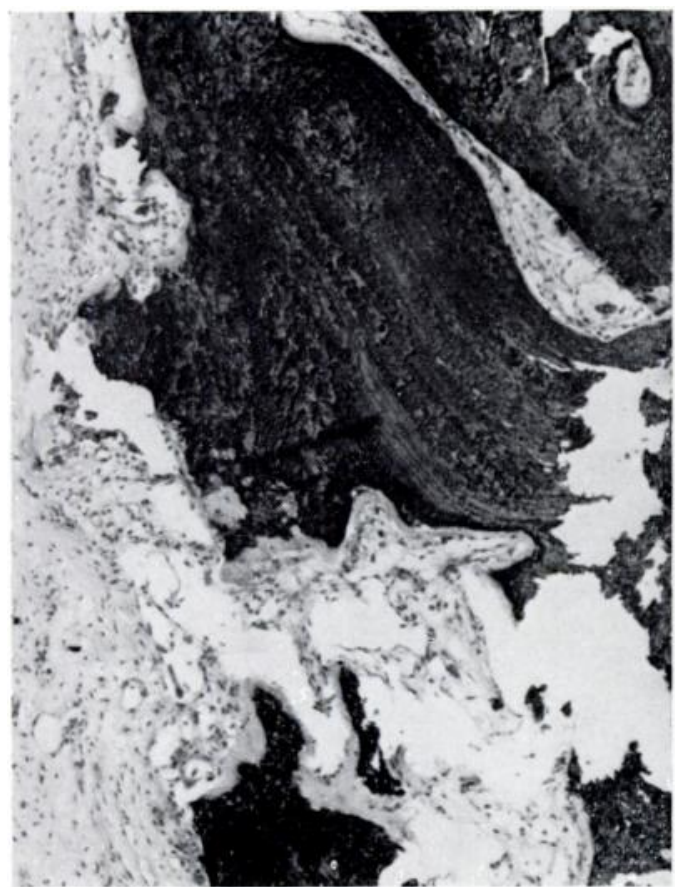

FIG. 21

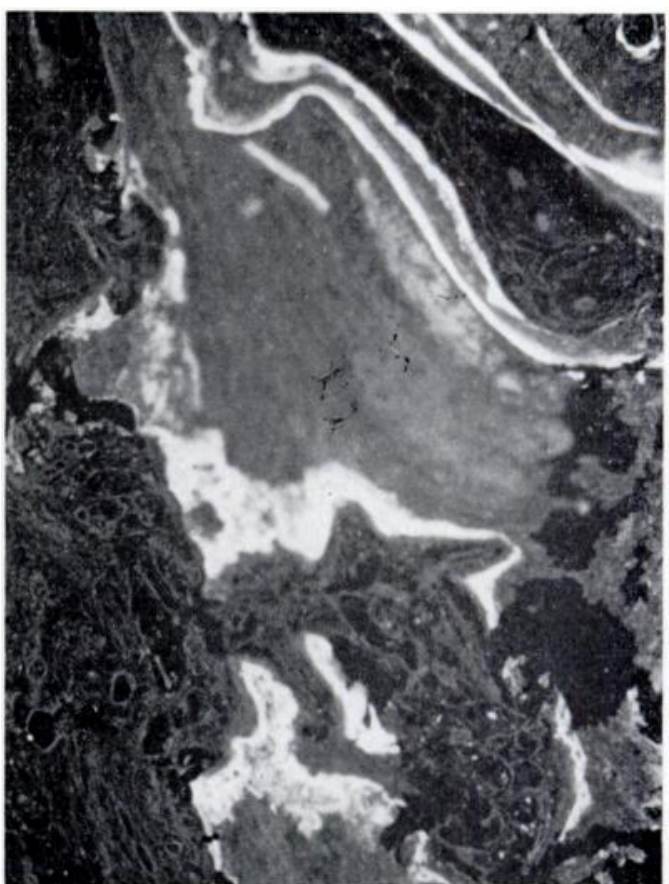

FIG. 22

Figure 21-Undecalcified section of bone adjacent to a bone cyst. Some distortion of the section has occurred during remounting after the ultra-violet examination shown in Figure 22. (Von Kossa stain, $\times 63$.) Figure 22The same section as in Figure 21 viewed with ultra-violet light before staining. Double labelling with tetracycline given twelve weeks and one week before operation. Appositional new bone has formed in the intervening period. $(\times 63$.

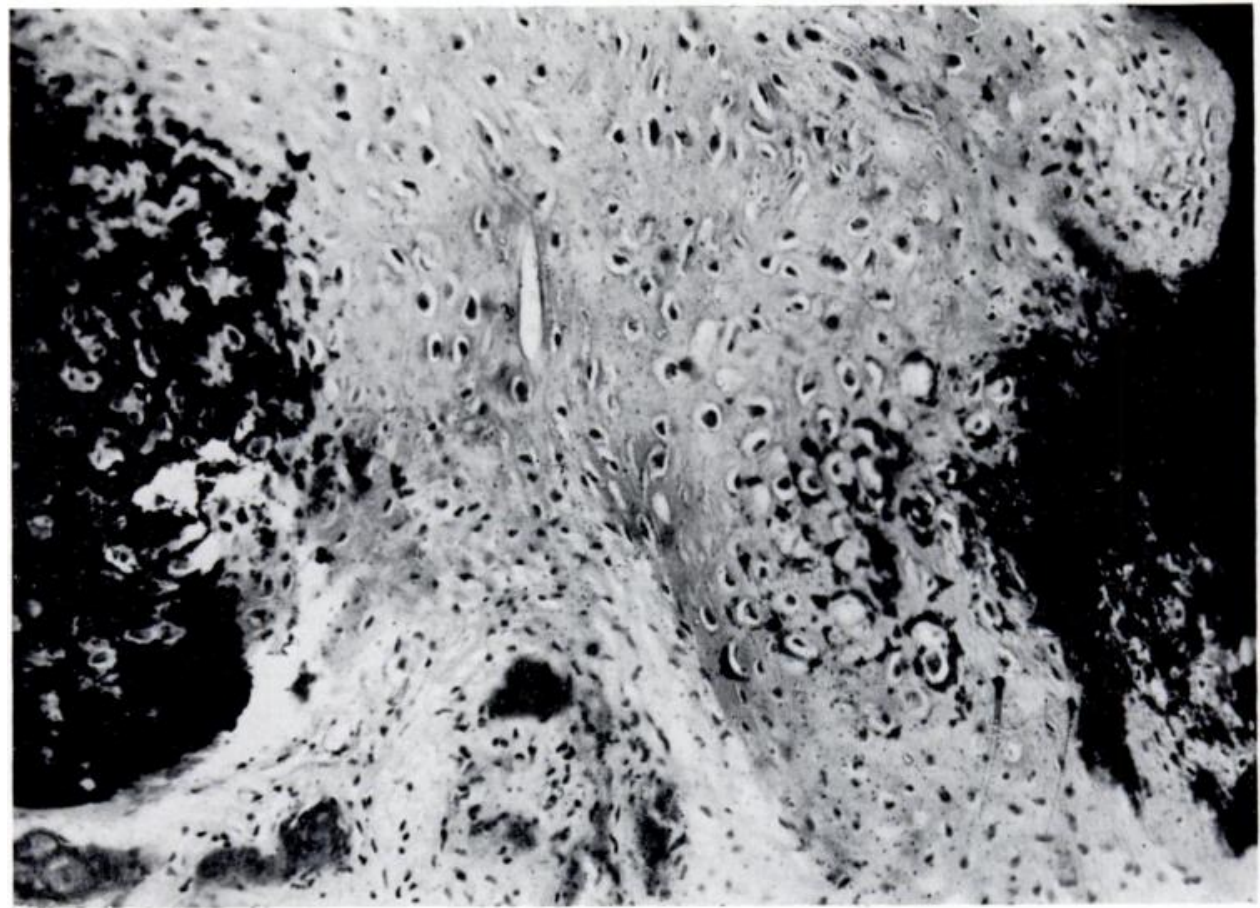

FIG. 23

Undecalcified section of a bone cyst showing cellular differentiation of fibrous tissue and enchondral new bone formation. (Von Kossa stain, $\times 174$.) 


\section{SUMMARY}

1. Osteogenesis in the osteoarthritic femoral head has been examined with radioactive ${ }^{32} \mathbf{P}$ and tetracycline bone markers.

2. In advanced osteoarthritis considerable osteogenic activity was observed, particularly in osteophytes, around cysts and in some areas of bone sclerosis.

3. Two forms of osteogenesis were seen: a form of enchondral ossification, and apposition of new bone to existing bone trabeculae.

4. The findings support previous studies suggesting that rapid turnover of bone tissues occurs in advanced osteoarthritis.

I wish to express my thanks to $\mathrm{Mr}$ B. Glynn-Jones who prepared the histological sections and to $\mathrm{Mr} \mathrm{B}$. C. Connor and $\mathrm{Mr} \mathrm{D}$. V. Weston for the photography. Mr H. D. Jamieson and Mr I. T. H. Ross gave valuable assistance with the isotope and autoradiographic studies.

\section{REFERENCES}

Ball, J. (1957): A Simple Method of Defining Osteoid in Undecalcified Sections. Journal of Clinical Pathology, $10,281$.

Batra, H. C., and Charnley, J. (1969): Existence and Incidence of Osteoid in Osteoarthritic Femoral Heads. Journal of Bone and Joint Surgery, 51-B, 366.

Bauer, G. C. H., and Smith, E. M. (1969): 85Sr Scintimetry in Osteoarthritis of the Knee. Journal of Nuclear Medicine, 10, 109.

Boyd, H. B., and Calandruccio, R. A. (1963): Further Observations on the Use of Radioactive Phosphorus (P32) to Determine the Viability of the Head of the Femur. Journal of Bone and Joint Surgery, 45-A, 445.

Collins, D. H. (1949): The Pathology of Articular and Spinal Diseases. London: Edward Arnold \& Co.

Harkison, M. H. M., Schajowicz, F., and Trueta, J. (1953): Osteoarthritis of the Hip: A Study of the Nature and Evolution of the Disease. Journal of Bone and Joint Surgery, 35-B, 598.

IbSen, K. H., and Urist, M. R. (1964): The Biochemistry and the Physiology of the Tetracyclines. Clinical Orthopaedics and Related Research, 32, 143.

Leblond, C. P., Wilkinson, G. W., Bélanger, L. F., and Robichon, J. (1950): Radio-autographic Visualization of Bone Formation in the Rat. American Journal of Anatomy, 86, 289. 